Apidologie, 1977, 8 (4), 437-449.

\title{
APHIDINA ALS HONIGTAUERZEUGER IN GÄRTEN UND PARKS MITTELEUROPAS MIT BESONDERER BERÜCKSICHTIGUNG DES BOTANISCHEN GARTENS FREIBURG/SCHWEIZ
}

\author{
Les Aphidiens, producteurs de miellat dans les jardins \\ et parcs d'Europe Centrale en considérant spécialement \\ le Jardin Botanique de Fribourg/Suisse
}

Gerolf LAMPEL

Entomologische Abteilung im Zoologischen Institut der Universität Freiburg/Schueiz

\section{SUMMARY}

APHIDINA AS HONEYDEW PRODUCERS IN GARDENS AND PARIS

OF GENTRAL EUROPE WITH SPECIAL REGARD TO THE BOTANIGAL GARDEN OF FREIBURG/SWITZERLAND

In the 18th century RÉaUmur detected honeydew to be the product of the defecation of plantlice and scale insects. Honeydew is used by different insects like bees, ants, wasps, bumble-bees, beetles, flies and so on. After a short discussion of the ecology of honeydew production and utilization special attention is directed to plantlice as honeydew producers in " artificial landscapes" (gardens and parks). While the so-called "wood-honeydew " is mainly a product of Lachnidae-Cinarinae, the honeydew in gardens and parks is produced also by other groups of aphids involving often a large number of species (see fig. $1+2$ and table 1 ). Some species may be important for the apiculture under special circumstances.

\section{ZUSAMMENFASSUNG}

Schon im 18. Jahrhundert erkannte RÉaumur, daß Honigtau ein Produkt der Defäkation von Blatt- und Schildläusen ist. Honigtau wird von verschiedenen Insekten wie Bienen, Ameisen, Faltenwespen, Hummeln, Käfern, Fliegen usw. genutzt. Nach einer kurzen Erörterung der Ökologie der Honigtauproduktion und -nutzung wird ein besonderes Augenmerk auf Blattläuse als Honigtauerzeuger in (c künstlichen Landschaften » (Gärten und Parks) gerichtet. 
Während der sogenannte « Waldhonigtau » vor allem ein Produkt von Lachnidae-Cinarinae ist, wird der Honigtau in Gärten und Parks auch von anderen Blattlausgruppen produziert, wobei oft eine große Zahl von Arten beteiligt sein kann (siehe Abb. $1+2$ und Tab. 1). Einige Arten können unter besonderen Umständen für die Bienenwirtschaft von Bedeutung sein.

Bereits im 18. Jahrhundert entdeckte Réaumur, daß Honigtau ein Produkt der Defäkation v.a. von Blatt- und Schildläusen ist, was 1864 von NöRDLINGER und 1891 von BüsgeN definitiv bestätigt wurde. Übersichten über honigtauerzeugende Blattläuse (Aphidina) verdanken wir u.a. BraUn (1938), Schmutterer (1958), Wille (1962) sowie Kloft, Fossel und Schels (1965). Dabei wurden v.a. diejenigen Arten berücksichtigt, die bienenwirtschaftlich für die sog. “ Waldtracht » von Bedeutung sind. Daß daneben auch Ameisen, Faltenwespen, Hummeln, Käfer, Fliegen und andere Insekten vom BlattlausHonigtau profitieren, ist bekannt. Die Nutznießer nehmen den Honigtau entweder von Pflanzenteilen auf, wohin er durch Abschleudern oder Abspritzen (Kunkel, 1972, 1973) gelangt, oder direkt vom Anus der Blattläuse, wie z.B. Ameisen und Fliegen, wobei der Honigtau teilweise erst durch das bekannte “ Betrillern » der “ Melkkühe » hervorgelockt wird. Während für die meisten Nutznießergruppen nur oberirdisch frei oder allenfalls in offenen Gallen lebende Honigtauspender in Frage kommen, können die Ameisen auch unterirdisch lebende oder von ihnen selbst mit Erdwällen umgebene Blattlauskolonien ausbeuten. Einige Blattläuse - die sogenannten formicobionten Arten - sind direkt auf das Abnehmen des Kotes durch die Ameisen angewiesen (Traminae, Stomaphis, Symydobius pp.) und würden ohne Ameisen im eigenen Kot festkleben. Andere Arten sind zwar beim Fehlen von Ameisen noch zum Abschleudern oder Abspritzen befähigt, doch reduzieren sie diese Tätigkeit, wenn ihre Kolonien lebhaft von Ameisen belaufen werden. Blattläuse in geschlossenen Gallen (viele Pemphigidae und Adelgidae am Hauptwirt) kommen als Honigtaulieferanten für andere Insekten nicht in Frage. Sie lösen das Problem der Kotbeseitigung durch Einhüllen desselben in Wachs.

\section{Übersichtsschema :}

Aphidina - an oberirdischen Pflanzenteilen frei oder in \pm offenen Gallen lebend

- an oberirdischen Pflanzenteilen in geschlossenen Gallen lebend
-. Honigtau wird abgespritzt, abgeschleudert oder (selten) in Wachs eingehüllt (z.B. Prociphilus anf Esche, Phyllaphis fagi) und in Tröpfchenform auf Pflanzenteilen abgelagert, von wo er durch Bienen, Ameisen, andere Hymenoptera, Fliegen und Käfer aufgenommen wird

- Honigtau wird direkt vom Anus der Blattläuse abgenommen (durch Ameisen und Fliegen) (Trophobiose)

- Honigtau kann nicht von anderen Insekten verwertet werden, wird durch Einhïllen in Wachs unschädlich gemacht 
an unterirdischen Pflanzenteilen lebend oder von Ameisen mit Erdwällen umbaut
-- formicobionte Blattläuse : Zur Honigtauabnahme vom Anus auf Ameisenbesuch angewiesen

- formicophile Blattläuse : In der Regel mit Ameisen vergesellschaftet, aber nicht unbedingt von diesen abhängig

- formicoxene Blattläuse : Werden nur gelegentlich von Ameisen besucht; vor Verkleben durch Honigtau durch Wachs. kleid geschützt (ZwöLFER, 1958)

Während Waldhonigtau vorwiegend von Lachnidae, besonders den nadelholzbewohnenden Cinarinae, geliefert wird, verschiebt sich das Bild in Parks und v.a. in Gärten zugunsten anderer Blattlausgruppen, außerdem wird es wesentlich mannigfaltiger, da in künstlichen Arboreta und in Gärten (besonders botanischen) mit der Zahl der Pflanzenarten auch die Zahl der Blattlausarten gegenüber einem Wald oder Forst wesentlich ansteigt. So wurden z.B. von Eastop in den Kew Gardens (London) 146 Blattlausarten, vom Verfasser im relativ kleinen Botanischen Garten Freiburg/Schweiz (1,5 ha) sogar 157 Blattlausarten gezählt. Allerdings sind von diesen Arten nur wenige, im BGF ca. 35, bienenwirtschaftlich \pm von Bedeutung, und das auch nur bei Massenvermehrung, wenn das Honigtauangebot die Reizschwelle der samrelnden Bienen überschreitet und zum Annoncieren dicser Trachtquelle im Stock veranlaßt. Am ehesten dürfte Honigtau von Bienen in reinen Arboreta, von Hecken, Alleebäumen und aus Gärten mit viel Zier- und/oder Obstgehölzen angenommen werden; in Gärten, die nur krautige Pflanzen enthalten, ist Honigtau dem Blütennektar unterlegen. Hier wird der Blattlaus-Honigtau vorwiegend von Ameisen genutzt. Zur Illustration des Gesagten seien in Abb. 1 a-c die Blattlaus-Eigenfunde aus dem Botanischen Garten Freiburg/Schweiz in drei Kreisschemata, getrennt nach Blattläusen auf Nadelhölzern, Laubhölzern und Krautpflanzen + Gräscrn sowie ihre (mögliche) Nutzung durch Bienen und Ameisen dargestellt (die Angaben über Ameisenbesuch größtenteils nach eigenen Beobachtungen). Abb. 2 a zeigt die Verteilung der 35 bienenwirtschaftlich \pm bedeutsamen Blattlausarten des BGF auf die cinzelnen AphidinaFamilien, Abb. 2 b die der 69 von Ameisen genutzten. Unter den Aphididae sind es v.a. Vertreter der Unterfamilien Aphidinae und Anuraphidinae, die sich durch Ameisenbesuch auszeichnen.

In bezug auf die befallenen Pflanzen können wir die in den Gärten und Parks Mitteleuropas auftretenden Blattläuse in drei Kategorien einteilen :

1) Blattläuse und Pflanzen indigen.

2) Blattläuse sowohl auf einheimischen, als auch auf gebietsfremden Pflanzen anzutreffen, wobei sie auf letzteren ev. weniger Honigtau erzeugen, 


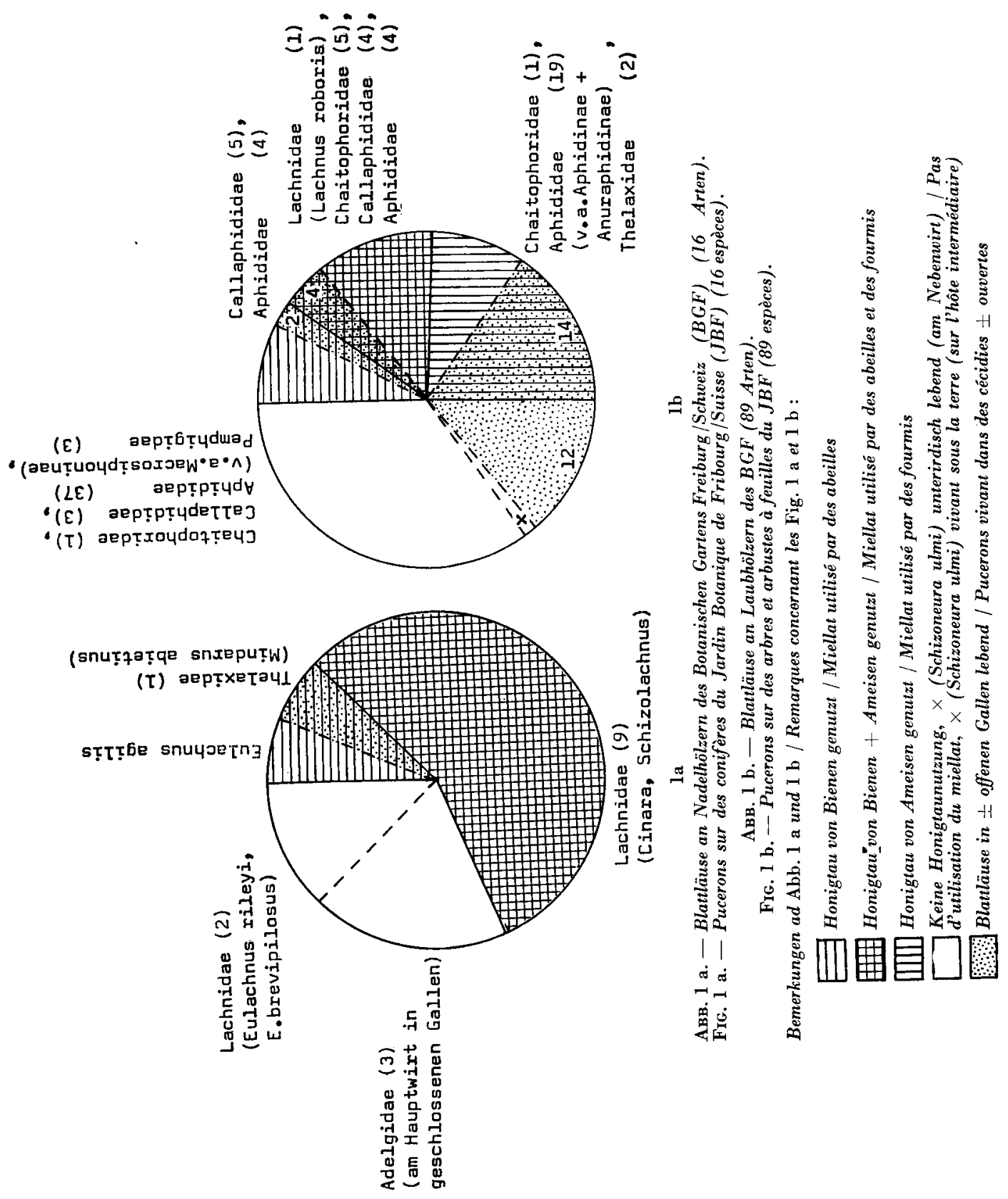




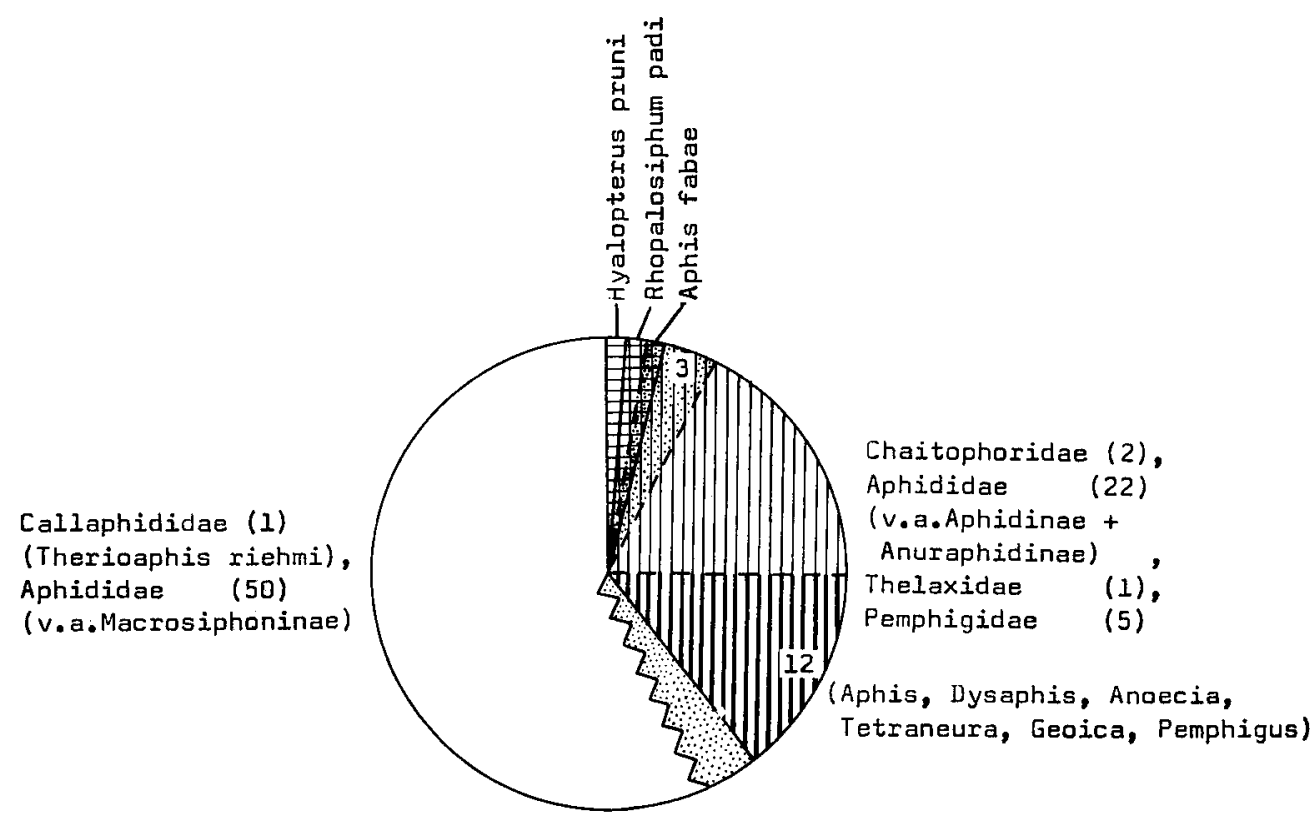

Авв. 1 c. - Blattläuse an Krautpflanzen und Gräsern des BGF (84 Arten).

Fig. 1 c. - Pucerons sur des plantes herbacées et des graminées du JBF (84 espèces).

Bemerkungen ad Abb. 1 c / Remarques ad Fig. 1 c :

Honigtau von Bienen genutzt / Miellat utilisé par des abeilles

册 Honigtau von Bienen + Ameisen genutzt / Miellat utilisé par des abeilles et des fourmis $\prod$ Honigtau von Ameisen genutzt, Blattläuse oberirdisch lebend / Miellat utilisé par des fourmis,

[1] Honigtau von Ameisen genutzt, Blatläuse unterirdisch lebend oder von Ameisen mit Erdwällen umbaut / Miellat utilisé par des fourmis, pucerons vivant sous la terre ou sous des levées de terre construites par les fourmis

Keine Honigtaunutzung / Pas d'utilisation du miellat

Blattläuse in \pm offenen Gallen lebend / Pucerons vivant dans des cécidies \pm ouvertes 
wie z.B. Beobachtungen von Maquelin (1974) an Cinara pectinatae (NördL.) zeigen.

3) Blattläuse mit ihren Wirtspflanzen aus fremden Gebieten eingeschleppt (selten).

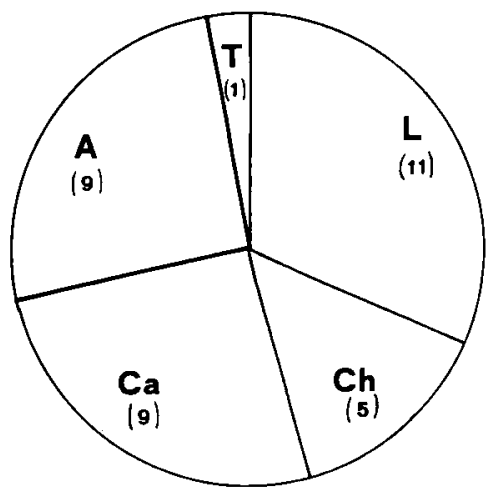

$\mathbf{a}$

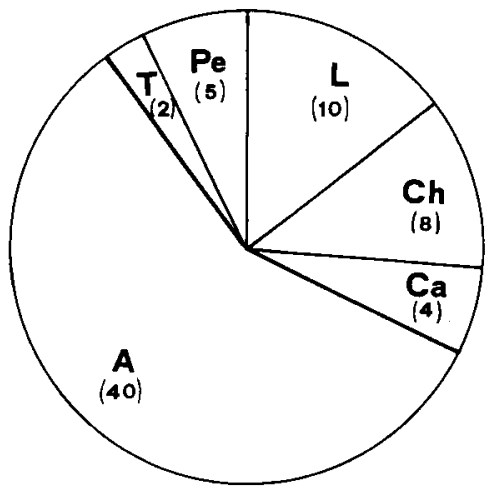

b

Авв. 2. - Die familienmäßige Aufteilung der honigtauerzeugenden Aphidina des BGF. a. - Bienenwirtschaftich \pm bedeutsame Arten.

b. - Von Ameisen genut:te Arten.

FIG. 2. - La répartition en familles des pucerons producteurs de miellat du JBF.

a. - Espèces d'un certain intêrêt pour l'apiculture.

b. - Espèces utilisées par des fourmis.

L Lachnidae, Ch Chaitophoridae, Ca Callaphididae, A Aphididae, T Thelaxidae, Pe Pemphigidae

Aus der großen Fülle (vergl. Tab. 1) seien nur einige Beispiele herausgegriffen :

Lachnidae : Die an der einheimischen Weißtanne Abies alba MrLL. vorkommende wichtige Honigtauerzeugerin Cinara pectinatae (NördL., 1880) ist in Parks auch an folgenden Tannen anzutreffen : Abies bornmuelleriana MAtTF. (Heimat : Kleinasien), A. nordmanniana (Stev.) Spach (Heimat : Kaukasus, Türkei), A. pinsapo Borss. (Heimat : S-Spanien.) Sie wurde von Maquelin (1974) mit positivem Erfolg auch auf junge Exemplare von $A$. concolor (GorD.) Hild. (Heimat : Nordamerika), A. koreana Wils. (Heimat : Korea) und A. veitchii Lindl. (Heimat : Japan) übertragen. - Nur an künstlich angepflanzten ausländischen Koniferen lebt die wahrscheinlich aus Nordamerika eingeschleppte Cinara cupressi (Bскт., 1881), und zwar an Thuja occidentalis L., Cupressus spp. und Juniperus spp. - Cinara brauni CB., 1940 wurde aus Südeuropa mit der Schwarzkiefer, Pinus nigra Ars., eingeführt, auf welche auch Cinara pini (L., 1758) übergehen kann. - Nur im natürlichen Biotop (vom Verf. z.B. im Aletschwald und Turtmanntal, Wallis, CH, gefunden), 
niemals in Gärten und Parks, ist Cinara cembrae (Chol., 1892) anzutreffen; auch der Verf. suchte sie vergebens an den zahlreichen Zirbelkiefern des BGF.

Chaitophoridae : Der wichtigste Honigtauerzeuger Periphyllus testudinaceus (FERN., 1852) wurde im BGF außer auf mitteleuropäischen auch auf folgenden Acer-Arten angetroffen : A. ginnala Maxim. (Heimat : Ostasien), A. negundo L. (Heimat : O-Nordamerika), A. opalus Milc. (Heimat: S-Europa), A. palmatum Thunb. (Heimat : Japan, Korea) und A. saccharum Marsh. (Heimat : Nordamerika); am Zuckerahorn fand sich auch Periphyllus acericola (WALK., 1848), am Fächerahorn P. aceris (L., 1761).

Callaphididae : Hier ist v.a. die südeuropäische Zierlaus Tuberculatus eggleri CB., 1950 zu nennen, die mit ihrer Wirtspflanze Quercus pubescens WILLD. nach M-Europa eingeschleppt wurde (auch vom Verf. im BGF gefunden). Auch Tuberculatus querceus (K ALT., 1843) und die bienenwirtschaftlich bedeutsame Art Tuberculoides annulatus (НтG., 1841) können an Q. pubescens übergehen (das gleiche gilt nach Funden des Verf. auch für die Lachnide Lachnus roboris (L., 1758)).

Aphididae : Viele Aphididae an Prunoideae und Pomoideae gehen in Gärten und Parks von einheimischen auch an ausländische Vertreter dieser Pflanzengruppen über, z.T. sind ihre (Haupt-) Wirte alte, schon früh aus Asien usw. nach M-Europa eingeführte Kulturpflanzen (Pfirsich, Mandel, Aprikose, Quitte). - Aphis fabae Scop., 1763 wurde im BGF an folgenden ausländischen Gehölzen angetroffen : Euonymus hamiltonianus Wall. (Ostasien), Cercis siliquastrum L. (S-Europa), Xanthoxylum bungei Planch. (China). - Eine typische Laus in Arboreta ist Acyrthosiphon caraganae (CHOL., 1907) von Caragana spp. und Colutea spp. - Zahlreich sind im BGF auch Beispiele für den Übergang von Aphididae von einheimischen auf ausländische Krautpflanzen.

Thelaxidae : Mindarus abietinus KocH, 1854, eine der wenigen Maskenläuse, deren Honigtau von Bienen genutzt wird, kommt außer an Abies alba MiLl. in Gärten und Parks auch an A. balsamea (L.) Mrll. (Nordamerika), A. bornmuelleriana MattF., A. concolor (Gond.) Hild., A. nordmanniana (Stev.) Spach und A. sachalinensis Mast. (N-Japan, Sachalin, Kurilen) vor (Angaben über Vorkommen auf Abies sibirica LEDEB. in $\mathbf{G}+\mathbf{P}$ dürften nach MitchelL, 1975, auf Fehldeterminationen beruhen).

Eingegangen im Mai 1977. Reçu pour publication en mai 1977.

\section{RÉSUMÉ}

Au Xviri ${ }^{e}$ siècle, RÉaumur a découvert que le miellat est un produit de la défécation des pucerons et des cochenilles. Le miellat est utilisé par divers insectes comme les abeilles, les fourmis, les guêpes, les bourdons, les coléoptères, les mouches etc. Après une brève discussion 
sur l'écologie de la proảuction et l'utilisation du miellat, on considère principalement les pucerons comme producteurs de miellat dans des (" paysages artificiels " (jardins, parcs). Contraitement au " miellat de forêt ", qui est un produit provenant surtout des LachnidaeCinarinae, le miellat des jardins et parcs est produit aussi par d'autres groupes de pucerons souvent présents en grand nombre d'espèces (voir fig. $1+2$ et tableau 1). Quelques espèces peuvent être importantes pour l'apiculture dans des circonstances spéciales.

La relation entre plantes et pucerons dans les jardins et pares d'Europe Centrale peut être divisée en 3 catégories :

1) Pucerons et plantes indigènes.

2) Pucerons vivant sur des plantes indigènes et des plantes introduites également.

3) Pucerons introduits avec leurs plantes hôtes de l'étranger (cas rare).

Sur le tableau 1, les plantes introduites sont spécialement indiquées. Au cas $n^{0} 3$ correspondent les espèces Cinara brauni CB., 1940, C. cupressi (ВСКT., 1881), Tuberculatus eggleri CB., 1950 et Acyrthosiphon caraganae (Сноц., 1907).

\section{LITERAT UR}

Braun R., 1938. - Die Honigtaufrage und die honigtauliefernden Kienläuse (Cinarini C.B.). Z. angew. Entomol., 24, 4.61-510.

Büsgen M., 1891. - Der Honigtan. Jenaische Z. Naturwiss., 25, 339-428.

EASTOP V. F., 1962-1963; 1965. - Additions to the wild fauna and flora of the Royal Botanic Gardens, Kew. XXV. A contribution to the aphid fauna. Kew Bull., 16, 139-146; XXVI. A second contribution to the aphid fauna. Kew Bull., 19, 391-397.

Kroft W., Fossel A. und Schels J., 1965. - Die Honigtau-Erzeuger des Waldes. In : Kloft W., Maurizio A. und Kaeser W. Das Waldhonigbuch. Ehrenwirth, München.

Kunkel H., 1972. - Die Kotabgabe bei Aphiden (Aphidina, Hemiptera). Bonn. zool. Beitr., 23, 161-178.

Kunkel H., 1973. - Die Kotabgabe von Aphiden (Aphidina, Hemiptera) unter Einfluß von Ameisen. Bonn. zool. Beitr., 24, 105-121.

Lampel G., 1974; 1975; 1976. Die Blattläuse (Aphidina) des Botanischen Gartens Freiburg/ Schweiz. Bull. Soc. Frib. Sc. Nat., 63, 59-137; 64, 125-184; 65.

MaQ̨uelin C., 1974. - Observations sur la biologie et l'écologie d'un puceron utile à l'apiculture : Buchneria pectinatae (NördL.) (Homoptera, Lachnidae). Diss. Eтr Zürich.

Mrtchell A., 1975. — Die Wald- und Parkbäume Europas. Parey : Hamburg und Berlin.

Nördulnger H., 1864. - Waldhonigthau. Krit. Blätter Forst- u. Jagdwiss., 46, 128-137.

Schmutterer H., 1958. - Die Honigtauerzeuger Mitteleuropas. Z. angew. Entomol., 42, 409419.

Wille H., 1962. - Le problème des miellats de forêt. J. Suisse apic., 59, 13-17, 40-48, 72-80, 100-104, 139-144.

ZwöLfer H., 1958. - Zur Systematik, Biologie und Ökologie unterirdisch lebender Aphiden (Homoptera, Aphidoidea), Teil IV (Ökologische und systematische Erörterungen). Z. angew. Entomol., 43, 1-52. 
TAB. 1. - Ubersicht über die wichtigsten von Bienen und Ameisen genutzten honigtauerzeugenden Blattlüuse in Gärten und Parlss Mitteleuropas mit besonderer Berücksichtingung des Botanischen Gartens Freiburg/Schweiz.

TABL. 1. - Vue d'ensemble des pucerons producteurs de miellat les plus importants utilisés par des abeilles et des fourmis dans les jardins et parcs d'Europe Centrale en considérant spécialement le Jardin Botanique de Fribourg/Suisse.

$\mathbf{n}=$ für die $\mathrm{CH}$ neue Art / espèce nouvelle pour la Suisse, ${ }^{*}=\mathrm{im} \mathrm{BGF}$ gefunden / trouvé dans le JBF, - = Eigenbeobachtung / observation personnelle.

Lachnidae E = unter von Ameisen errichteten Erdwällen / sous des levées de terre construites par des fourmis, $\mathrm{U}=$ unterirdisch / sous Ia terre

n * Cinara brauni CB., 1940

Cinara confinis (KocH, 1865)

Cinara costata (ZetT., 1828)

* Cinara cuneomaculata

(d. Gu., 1909)

n * Cinara cupressi (Bскт., 1881)

* Cinara juniperi (d. G., 1773) Cinara kochiana (CB., 1939)

Cinara laricis (Hтg., 1839)

Cinara pectinatae

(Nörol., 1880)

Cinara piceae (Panz., 1801)

* Cinara pilicornis (HTG., 1841)

* Cinara pinea (MoRow., 1895)

* Cinara pini (L., 1758)

Cinara pruinosa (HTg., 1841)

n * Cinara stroyani (PǍ̌sK, 1954)

* Eulachnus agilis

(KaLT., 1843)

Lachnus pallipes

(HTG., 1841)

- Lachnus roboris (L., 1758)

Maculolachnus submacula

(WALK., 1848)

- Schizolachnus pineti

(F., 1781)

Stomaphis quercus (L., 1758)

Stomaphis longirostris

(F., 1787)

Tuberolachnus salignus

(GMEL., 1790)
Wirtspflanzen, - - - -

in ME nicht einheim.,

in $G+P$ angepflanzt

Plantes hôtes, - - -

mais plantées dans les jardins et parcs

Pinus nigra ARN. -

Ábies alba MrLL., A. cilicica

CARR., A nordmanniana SPACH,

A.sachalinensis MAst.

Picea abies KARst., $P$ glauca

Voss, $P$. mariana B.S.P., $P$.

omorika PURKYNE, $P$. sitchensis

CÁR.

Larix decidua MiLl., L. kaemp:

feri CarR.

Thuja occidentalis L. •, Cupressus spp., Juniperus spp.

Jüniperus communis $\mathrm{L}$.

Larix decidua MiLL., L. kaemp:

feri CARR.

Larix decidua MiLL., L. kaemp-

feri CARR.

Abies alba Mill., $A$. bornmuelleriana MATTF., $A-$ nord manniana SPACH, $A$ pinsapo Boiss., weitere Schmucktannen

Picea abies KARST.

Picea abies KARST., P. sitchensis

CARR.

Pinus silvestris $\mathrm{L} ., \quad P$. mugo

TURRA -

Pinus silvestris L., P. mugo

TuRra, $P$ nigra ARN.

Picea abies KARst., $P$ Klauca Voss

Picea abies KARst.

Pinus silvestris L., P. mugo Turra -

Fagus silvatica $\mathbf{L}$.

Quercus petraea (MАтT.) LiEBL. $Q$. robur L., $Q$._pubescens Willd. •, Castanea sativa MiĹ. Rosa spp.

Pinus silvestris L., P. mugo Turra

Quercus spp.

Populus spp., Salix spp.

Salix spp.

\begin{tabular}{|c|c|c|}
\hline $\begin{array}{l}\text { Anm. } \\
\text { Rem. }\end{array}$ & $\begin{array}{c}\text { D. Bienen } \\
\text { genutzt } \\
\text { Utilisé } \\
\text { par des } \\
\text { abeilles }\end{array}$ & \begin{tabular}{|c} 
D. Ameisen \\
genutzt \\
Utilisé \\
par des \\
fourmis
\end{tabular} \\
\hline \multirow[t]{2}{*}{ pp. U } & $\begin{array}{l}+ \\
+\end{array}$ & + \\
\hline & + & $(+)$ \\
\hline \multirow{4}{*}{ pp. $\mathbf{U}$} & $\begin{array}{l}+ \\
+\end{array}$ & $\begin{array}{c}+ \\
(+)\end{array}$ \\
\hline & $\begin{array}{l}+ \\
+\end{array}$ & + \\
\hline & ++ & + \\
\hline & +++ & $(+)$ \\
\hline \multirow[t]{3}{*}{$\begin{array}{l}\text { pp. } \mathrm{U} \\
\text { pp. E }\end{array}$} & + & $\stackrel{+}{+}$ \\
\hline & + & $+\cdot$ \\
\hline & + & -++ \\
\hline pp. U & $(+)$ & + \\
\hline$\underset{(?)}{\text { pp. } U}$ & $\begin{array}{c}t \\
(+)\end{array}$ & ++ \\
\hline pp. U & $(+)$ & + \\
\hline \multirow{5}{*}{ pp. U } & + & ++ \\
\hline & & + \\
\hline & + & + \\
\hline & & $\begin{array}{c}++ \\
+\end{array}$ \\
\hline & + & + \\
\hline
\end{tabular}

Anmerkungen : Nicht in G + P, nur im natürlichen Biotop : Cinara combrae (CHoL., 1892) (vergl. LAMPEL, 1974), Cinara noubergi (ARnir.. 1930).

Pinus mugo TunRa : Durcligehende Unterstreichung bedeutet : Nur in $G+P$ befallen

Remarques : Absent des J + P, seulement dans le biotope naturel : Cinara cembrae (CHoL., 1892) (voir LAmpeL, 1974), Cinara neubergi (ARNH., 1930)

Pinus mugo Turfa : Soulignement sans interruption signifie : Attaquée seulement dans les jardins et parcs 
Chaitophoridae Ae Aestivosistentes.

\begin{tabular}{|c|c|c|c|c|}
\hline $\begin{array}{c}\text { Blattläuse } \\
\text { Pucerons }\end{array}$ & $\begin{array}{l}\text { Wirtspflanzen, }---- \\
\text { in } M E \text { nicht einheim., } \\
\text { in } \mathbf{G}+\mathbf{P} \text { angepflanzt } \\
\text { Plantes hôtes, }--- \\
\text { non indigène en EC, } \\
\text { mais plantée dans les jardins et parcs }\end{array}$ & $\begin{array}{l}\text { Anm. } \\
\text { Rem. }\end{array}$ & $\begin{array}{l}\text { D. Bienen } \\
\text { genutzt } \\
\text { Utilisé } \\
\text { par des } \\
\text { abeilles }\end{array}$ & $\begin{array}{l}\text { D. Ameisen } \\
\text { genutzt } \\
\text { Utilisé } \\
\text { par des } \\
\text { fourmis }\end{array}$ \\
\hline $\begin{array}{l}\text { * Chaitophorus popublialbae } \\
\text { (B.d.F., 1841) } \\
\text { * Chaitophorus populeti } \\
\text { (PANz., 1805) } \\
\text { * Periphyllus acericola } \\
\text { (WALK., 1848) } \\
\text { Periphyllus aceris (L., 1761) } \\
\text { Periphyllus coracinus } \\
\text { (Koch, 1854) } \\
\text { Periphyllus hirticornis } \\
\text { (WALK., 1848) } \\
\text { Periphyllus lyropictus } \\
\text { (KESsLER, 1886) } \\
\text { Periphyllus testudinaceus } \\
\text { (FERN., 1852) }\end{array}$ & 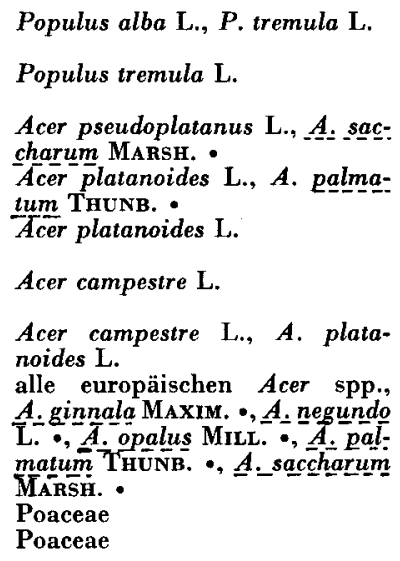 & $\begin{array}{l}\text { Ae } \\
\text { Ae }\end{array}$ & $\begin{array}{c}+ \\
(+) \\
(+) \\
+ \\
(+) \\
++ \\
++\end{array}$ & $\begin{array}{l}+ \\
+ \\
+ \\
+ \\
+ \\
+ \\
+ \\
+\end{array}$ \\
\hline
\end{tabular}




\begin{tabular}{|c|c|c|c|c|}
\hline $\begin{array}{c}\text { Blattläuse } \\
\text { Pucerons }\end{array}$ & $\begin{array}{c}\text { Wirtsp flanzen, - - } \\
\text { in } M E \text { nicht einheim., } \\
\text { in } G+P \text { angepflanzt } \\
\text { Plantes hôtes, }--- \\
\text { non indigène en EC, } \\
\text { mais plantée dans les jardins et parcs }\end{array}$ & $\begin{array}{l}\text { Anm. } \\
\text { Rem. }\end{array}$ & $\begin{array}{l}\text { D. Bienen } \\
\text { genutzt } \\
\text { Utilisé } \\
\text { par des } \\
\text { abeilles }\end{array}$ & $\begin{array}{l}\text { D. Ameisen } \\
\text { genutzt } \\
\text { Utilisé } \\
\text { par des } \\
\text { fourmis }\end{array}$ \\
\hline 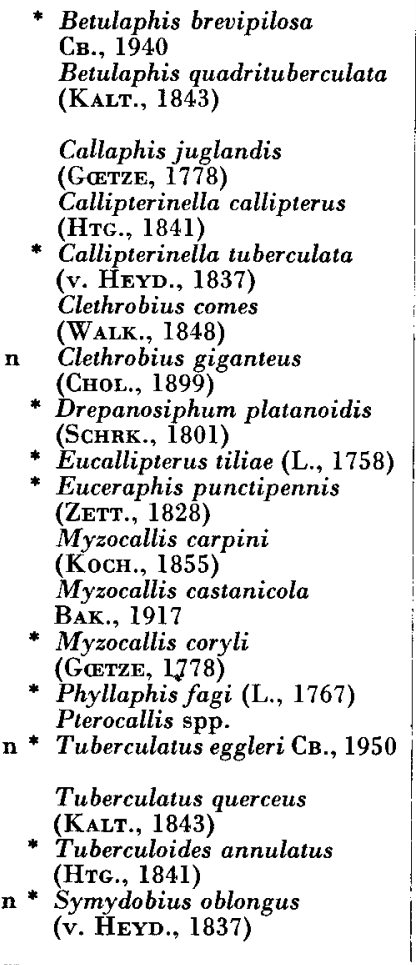 & 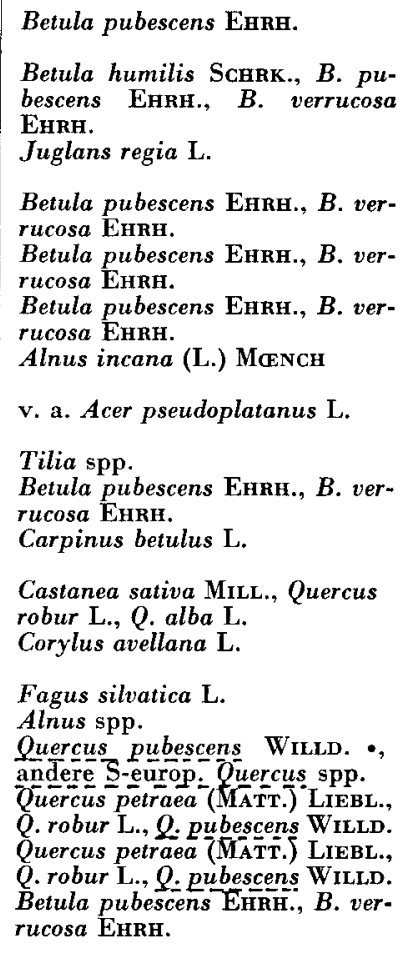 & G & $\begin{array}{c}(+) \\
(+) \\
(+) \\
+ \\
+ \\
(+) \\
+ \\
(+) \\
+ \\
+ \\
+ \\
+ \\
(+) \\
+ \\
+ \\
++ \\
(+)\end{array}$ & $\begin{array}{c}+ \\
+ \\
+ \\
(+) \\
(+)\end{array}$ \\
\hline
\end{tabular}


Aphididae E unter von Ameisen errichteten Erdwällen / sous des levées de terre construites par des fourmis, $\mathrm{G}$ offene Gallen / cécidies ouvertes, HW Hauptwirt / hôte principal, NW Nebenwirt / hôte intermédiaire, U unterirdisch / sous la terre.

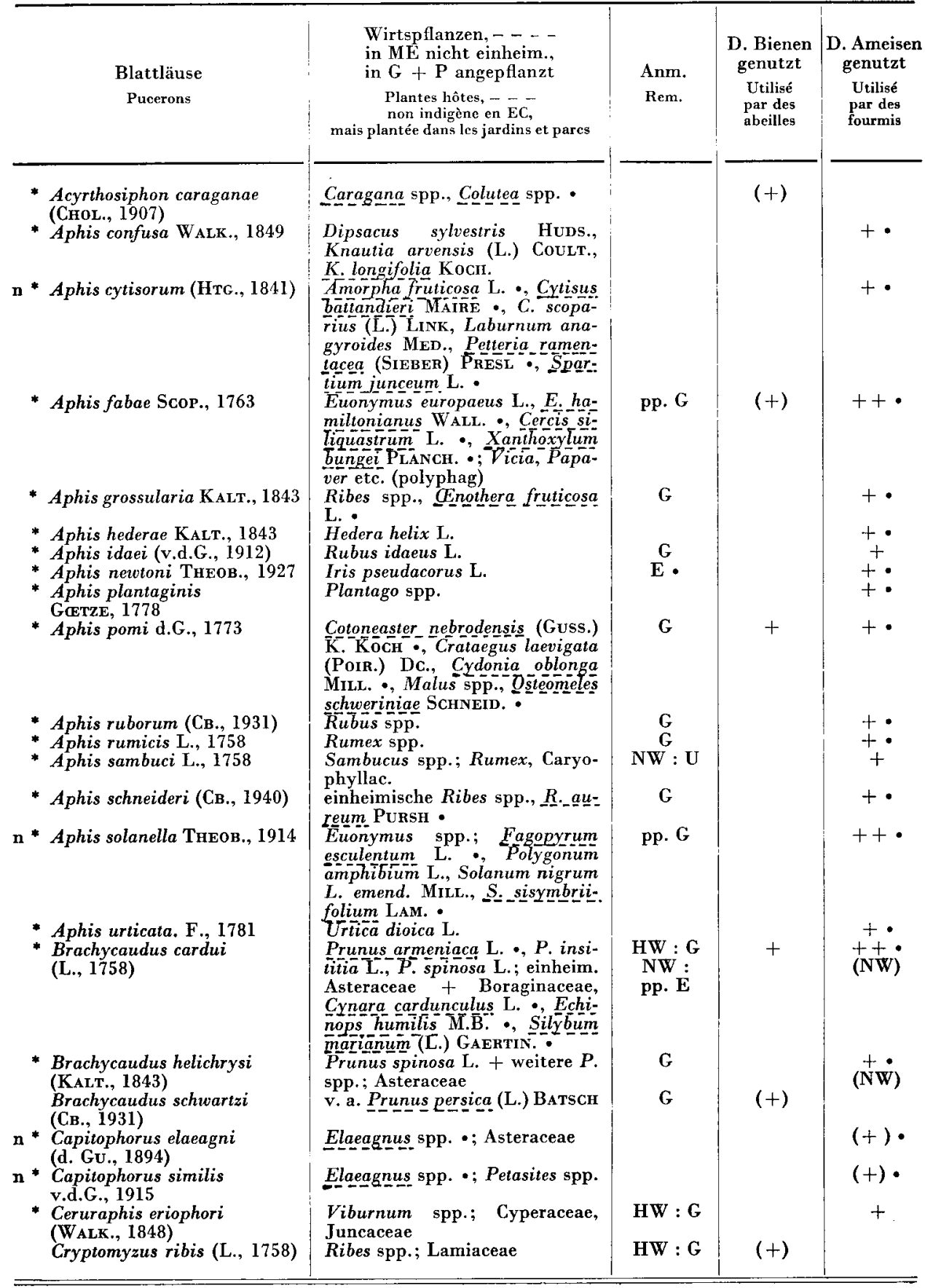


Aphididae, Fortsetzung $\mathbf{E}$ unter von Ameisen errichteten Erdwällen / sous des levées de terre construites par des fournis; G offene Gallen / cécidics ouvertes, HW Hauptwirt / hôte principal, NW Nebenwirt / hôte intermédiaire, $\mathrm{U}$ unterirdisch / sous la terrc.

n * Dysaphis angelicae (Kocil, 1854)

* Dysaphis anthrisci Cв., 1950

* Dysaphis crataegi

(KALT., 1843)

* Dysaphis lauberti (Св., 1940)

- Dysaphis petroselini (CB., 1950)

* Dysaphis plantaginea (Pass., 1860)

n * Dysaphis ranunculi (Kalt., 1843)

Hyalopterus amygdali (Blanch., 1840)

* Hyalopterus pruni (Geoffr., 1762)

* Macrosiphum rosae (L., 1758)

n * Metopcurum fuscoviride Stroyan, 1950

* Metopolophium dirhodum (W ALK., 1849)

* Myzus cerasi (F., 1775)

* Myzus lythri (SchRк., 1801)

* Myzus persicae (SULz., 1776)

* Pterocomma populeum (KALT., 184.3)

Pterocomma salicis (L., 1758)

* Rhopalosiphum insertum (WALK., 1848)

* Rhopalosiphum maidis (Fitch, 1856)

* Rhopalosiphum nymphaeae (L., 1761)

* Rhopalosiphum padi (L., 1758)

n * Schizaphis typhae (LAING, 1923)

n * Uroleucon tussilaginis (WALK., 1850)

* Vesiculaphis theobaldi TAK., 1930
Wirtspflanzen, - - - in ME nicht einheim., in $G+P$ angepflanzt

Plantes hôtes, -. - -

non indigène en EC. mais plantée dans les jardins et pares

Crataegus spp., Cotoneaster simonsii BAKEn : Angelica archangelica $\mathrm{L}$.

Malus fusca (Raf.) Schneid. •, weitere Malus spp.; Anthriscus sylvestris (L.) HOFFM.

Crataegus spp.; Daucus carota $\mathbf{L}$.

Crataegus spp., Cotoneaster simonsii BAKER -; Heracleum sphondylium I., H. mantegazzianum So et LEv.

Trataegus spp.; Apiaceac

Malus spp.; Plantago spp.

Crataegus spp.; einheimische Ranunculus spp., $\underline{R}$. muricatus L. •

Prunus persica (L.) Batscil; Phragmites, M̄olinia

Prunus spinosa L., P. domestica L.; Phragmites communis Trin., Arundo donax L. •, Typha spp.

Rosa spp.; Dipsacaceae

Tanacetum vulgare $\mathrm{L}$.

Rosa spp.; Poaceac

Prunus avium L., P. cerasus L., $P$. mahaleb L., P. cerasifera. Eнrн. •; Galium spp., Veronica spp.

Pruntus mahaleb L.; Lythrum spp.

Prunus persica (L.) BATSCH $\bullet$, P. amygdalus BATSCH •; sehr polyphag an Krautpflanzen Populus spp.

Salix spp.

einheim. Pomoideae, Cotoneasster spp. •, Cydonia oblonga Mill. Malus spp. Foaceae Zea mays L. , andere Poaceac

einheim. Prunus spp., $\boldsymbol{P}_{-}$cera: sifera Eirkh. -; Sumpfpfilanzen Pruñus padus L., ausl. Prunus spp. •; Poaceae

Typha spp.

Tussilago farfara L., Petasites spp.

Carex spp., Schoenus nigricans L.
D. Bienen D. Ameisen

Anm. genutzt genutzt

Rem.

Utilisé abeilles pardes

HW : G

NW : U

(N)

IIW : G

NW : U

IIW : G

$\mathrm{NW}: \mathrm{U}$

IIW : $\mathrm{G}$

NW : U

$+\mathbf{E}$.

IIW : G

$\mathrm{NW}: \mathrm{U}$

$+\mathbf{E}$.

IIW : $\mathrm{G}$

NW : U

HW : G

$\mathrm{NW}: \mathrm{U}$

$+\mathbf{E}$.

(NW)

(NW)

(NW)

(NW)

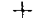

+ .

(+)

(t)

(t) •

(NW)

+ -

(+) •

IIW : G

(HW)

(HW)

HW : G

(HW)

IIW : G

(+) (HW)

IIW : G

(+)

NW: U

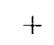

$+$

$+$

(HW)

+ .

HW : G

$+$

(t) 
Thelaxidae G offene Gallen / céeidies ouvertes, NW Nebenwirt / hôte intermédiaire, U unterirdisch / sous la terre

\begin{tabular}{|c|c|c|c|c|}
\hline $\begin{array}{c}\text { Blattläuse } \\
\text { Pucerons }\end{array}$ & $\begin{array}{c}\text { Wirtspflanzen, } \\
\text { in } \mathrm{ME} \text { nicht einheim., } \\
\text { in } \mathrm{G}+\mathrm{P} \text { angepflanzt } \\
\text { Plantes hôtes, }--- \\
\text { non indigène en } \mathrm{EC}, \\
\text { mais plantée dans les jardins et parcs }\end{array}$ & $\begin{array}{l}\text { Anm. } \\
\text { Rem. }\end{array}$ & $\begin{array}{c}\text { D. Bienen } \\
\text { genutzt } \\
\text { Utilisé } \\
\text { par des } \\
\text { abeilles }\end{array}$ & $\begin{array}{c}\text { D. Ameisen } \\
\text { genutzt } \\
\text { Utilisé } \\
\text { par des } \\
\text { fourmis }\end{array}$ \\
\hline $\begin{array}{l}\text { * Anocia sp. der A. corni- } \\
\text { Gruppe Glyphina betulae } \\
\text { (L., 1758) } \\
\text { n * Hamamelistes betulinus } \\
\text { (Horv., 1896) } \\
\text { * Mindarus abietinus } \\
\text { Koch, } 1857\end{array}$ & 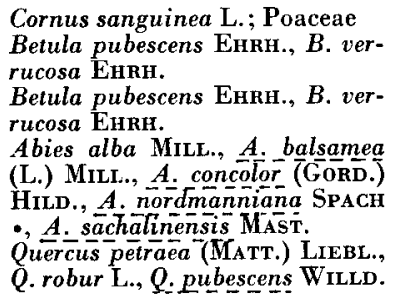 & $\mathbf{N W}: \mathbf{U}$ & $(+)$ & $\begin{array}{c}++ \\
+ \\
+\end{array}$ \\
\hline
\end{tabular}

Pemphigidae G offene Gallen / cécidies ouvertes, G geschlossene Gallen / cécidies fermées HW Hauptwirt / hôte principal, NW Nebenwirt / hôte intermédiaire, U unterirdisch / sous la terre

\begin{tabular}{|c|c|c|c|c|}
\hline $\begin{array}{c}\text { Blattläuse } \\
\text { Pucerons }\end{array}$ & $\begin{array}{l}\text { Wirtspflanzen, } \\
\text { in ME nicht einheim., } \\
\text { in } \mathbf{G}+\mathbf{P} \text { angepflanzt } \\
\text { Plantes hôtes, }-\overline{-}- \\
\text { non indigène en EC, } \\
\text { mais plantée dans les jardins et parcs }\end{array}$ & $\begin{array}{l}\text { Arun. } \\
\text { Rem. }\end{array}$ & $\begin{array}{l}\text { D. Bienen } \\
\text { genutzt } \\
\text { Utilisé } \\
\text { par des } \\
\text { abeilles }\end{array}$ & $\begin{array}{l}\text { D. Ameisen } \\
\text { genutzt } \\
\text { Utilisé } \\
\text { par des } \\
\text { fourmis }\end{array}$ \\
\hline $\begin{array}{l}\text { Baizongia pistaciae (L., 1767) } \\
\text { Forda spp. } \\
\text { n* Geoica eragrostidis } \\
\text { (PAss., 1860) } \\
\text { * Geoica setulosa (PAss., 1860) } \\
\text { * Pemphigus bursarius } \\
\text { (L., 1758) } \\
\text { Prociphilus bumeliae } \\
\text { (ScHRK., 1801) } \\
\text { Prociphilus fraxini (F., 1777) } \\
\text { * Schizoneura ulmi (L., 1758) } \\
\text { Smynthurodes betae } \\
\text { WESTW., 1849 } \\
\text { Tetraneura ulmi (L., 1758) } \\
\text { * Thecabius affinis } \\
\text { (KaLT., 1843) }\end{array}$ & 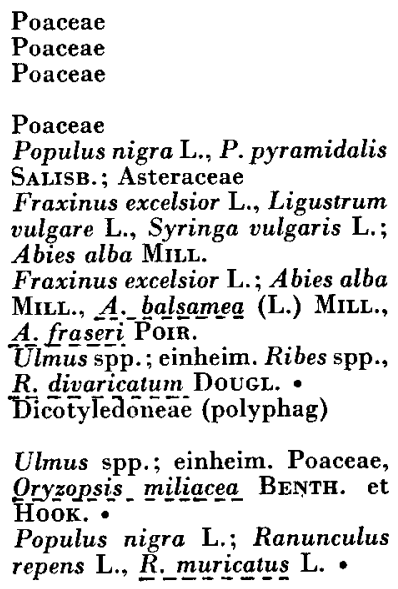 & 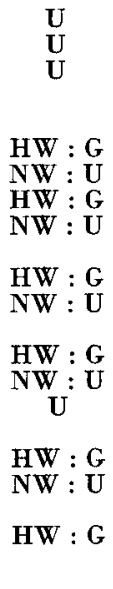 & $(+)$ & $\begin{array}{c}++ \\
++ \\
++ \\
++ \\
(+) \\
(\mathrm{NW}) \\
+ \\
+\end{array}$ \\
\hline
\end{tabular}

